\title{
Lauren J. Silver: System Kids: Adolescent Mothers and the Politics of Regulation
}

\author{
The University of North Carolina Press, Chapel Hill, NC, 2015, 198 pp, \\ ISBN: 9781469622590
}

\author{
Mikayla Flanagan ${ }^{1}$
}

Received: 8 June 2015/Accepted: 8 June 2015/Published online: 8 July 2015

(C) Springer International Publishing 2015

System Kids: Adolescent Mothers and the Politics of Regulation, by Lauren J. Silver, introduced new ideas to revamp the Supervised Independent Living program (SIL) by offering a clear understanding of the terror behind the scenes. She does so to reveal that the hierarchical order of government-provided services needs to be more encompassing; it essentially needs those of higher standing to become more involved in the actual provision of services and the functioning of programs. "Without adequate government support, marginalized adolescent mothers and their children are susceptible to greater hardship, and hence it is vital that the institutions and programs in place be reformed justly (not eliminated)" (Silver 2015, p. 20). That quote was the basis of the book, but every chapter contributed distinctive narratives in support of it. Silver explored the relationship distances between staff and clients, the neglect of better residential quarters, the stigmas and consequences that come with being an adolescent delinquent mother, while addressing the ways to cope with these problems.

In the first chapter, the author started detailing the various conditions in which the caseworkers have to work, as well as the frustrations that come with that type of environment. It came across as though the SIL set their employees and caseworkers for failure. The SIL had hierarchal issues within the staff because the program managers, business administrators, and supervisors did not experience the reality of the program. Instead, they had an illusion of what they felt transpired in these residential sites. Caseworkers' offices, in rundown areas in the inner

\footnotetext{
Mikayla Flanagan

mikshelt@indiana.edu

1 Indiana University, Bloomington, IN, USA
}

city, made use of secondhand furniture, which did not provide the ambiance of professionalism. On the other hand, the administrators had newly furnished offices in suburban areas. Additionally, the caseworkers were not equipped with adequate technology to perform their jobs effectively. In writing this chapter, Silver highlighted the existing hierarchical and social differences, undoubtedly signifying the inferior status of caseworkers in the organization. Stating this in the beginning helps to set the tone of the book as it becomes a prominent theme throughout the chapters.

The SIL program was funded by the child welfare agency, Children and Youth Services (CYS), to provide educational, residential, and social services for adolescent mothers, most of whom were delinquents. Each caseworker had an overabundance of clients, which ultimately limited their effectiveness. They were not able to provide an appropriate amount of attention to their clients on top of all the paperwork that they had to complete daily. The lack of professionalism and negligence of clients in their residential quarters caused the relationships to be unhealthy and strained. The clients failed to notice the stress caseworkers experienced and ultimately accused them of not caring, despite the leniency that extended. The thought that this provokes is very profound because one would think that these delinquent mothers came from broken homes of some sort. Yet, the government placed them in the SIL program, which does not provide adequate care and support for adolescents. The mothers were looking for some type of family in the SIL program but they did not seem to find it under the circumstances. This situation raises a compelling question: Are these types of programs actually helpful or are they enabling adolescent mothers to continue to do what they want without the needed guidance and assistance? 
In chapter two, the author presents how the youth and caseworkers had to grapple with the insufficiencies of the SIL residential environment. Being in these circumstances had caused both the caseworkers and mothers to compromise the rules just to make living in the dangerous setting less stringent and more durable. Familiar zones were used to hide the violence and the outsiders that lived with the participants, both of which were incompliant with the program. Instead of referencing policies for decisionmaking, administrators and those who are not on the ground should try to understand the familiar zones put in place in efforts to address the structural problems inflicted on the youth and caseworkers. A drug-related murder of a live-in boyfriend alarmed the adolescents and subsequently led to increased concern. The caseworkers and program managers could not afford to move every mother to another location, nor the entire location to a better part of town because no landlord wanted delinquents in their building. The program could not improve itself because of the stigma that these adolescent mothers carried when searching for better housing. The need and desire was there, but the opportunity was not.

The chapter also mentioned a client, Tomeka, who had to attend court because she was unable to finish school due to a lack of childcare. Tomeka's situation raised familiar questions. Why require school, but provide no childcare? How do they expect adolescent mothers to get a grasp on life without adequate childcare? In the court proceedings, the judge ordered her to go to the SIL's GED program. The judge was unaware that the girls in the GED program brought their children to class, which negatively impacted their education. Tomeka was a perfect example of the disconnect that exists between the clients and staff of the program. Silver again presented the theme that social realities do not match official descriptions; they did not push the mothers and caseworkers in the direction of sustainability. There is a conflict of interest embedded in the SIL program because administrators are supposed to promote a supportive, safe and independent lifestyle for the adolescents; yet, the system is building more adverse conditions, presenting solutions that are unrealistic, and maintaining the structure of the program with all of its inadequacies.

Chapter three examined how the stigmas hindered the development of the program and the adolescents. The adolescents faced issues of victimization, self-efficacy, and self-identity. Through case hearings, they had to explain why they felt worthy of public investment-yes, public investment. These young girls came into the system at young ages and had to prove that they would be successful once out of the program. The younger they were, the easier it was to get accepted to the program because of greater potential for positive influences. If the mothers were older (e.g., 17 years of age), they were viewed as being set in their ways, and the hope for a positive change was considered to be a waste of time and money. For example, Denise, a 17-year-old mother of a 3-year-old, had been homeless for over a year and was in the process of being evaluated for entrance into the SIL program. Denise decided to seek shelter because she had an elderly grandmother, whom she felt a burden to, and a mother who did not provide for Denise or her child. In all actuality, CYS wanted to save money by denying her services because of her age; this was even though her case was worse than other women in the SIL program.

The system accepts youth of various backgrounds and, simultaneously, holds them accountable for making mature decisions. The system has forgotten the mentality of adolescents. The author illustrated how these mothers used the chance for growth and development to manipulate the minds of the evaluators and gain pity. The adolescents made themselves appear needy and used their pasts to justify their delinquency. This effected how the caseworkers uniquely dealt with each case. Kelley had been moved around quite a bit and faced the possibility of being discharged and losing the custody of her child. She depicted herself as a victim and the caseworkers felt that she was simply telling them what they wanted to hear. They asked her to leave and deliberated without her being present. A similar case occurred, but this particular mother characterized herself efficaciously. The caseworkers believed she was more than capable of fixing her issues. She was able to stay and hear their constructive criticism.

As the adolescent mothers continued to discover themselves, Silver explained how their new-formed identities could affect their performances as youth. Chapter 4 illustrated this well by showing how the system caused the youth to exploit themselves in order to get further assistance. The author shadowed a client to understand the tribulations of adolescent mothers. She saw how privileged people could be taken seriously. However, the stigma that these adolescents faced restricted them from having a fair chance in society because the system did not take them seriously enough. The system has protocols and spatial barriers in place that forced youth to learn how to manage self-identity in a particular way depending on the person, in order to seem deserving.

Nyisha, a 21-year-old mother who was about to reach the maximum age in the system, could not find adequate housing because of a debt her mother put in her name when she was 10. Nyisha viewed herself as she felt others perceived her, an African-American victim that was not worth much. She recognized that the SIL program was a blessing because she would have been in jail without it. To be successful, she had to build some type of relationship with her caseworkers, which was not always possible due to 
structural problems. These types of relationships are critical in adolescent development. Since she was not taken seriously, she had to use different avenues and various people to receive the help she needed. The system forced Nyisha to reveal painful memories of being raped just to give her the run around. All she longed for was safe housing and recounting her past was the only way of justifying the necessity. Nyisha's circumstances raise so many complex questions. Is that not unjust? Was she just asking for too much? Regardless of how this comes across, the government system has not budged on the procedure that they follow and could not accommodate her needs effectively.

Previous chapters focused on resistance of protocols and program expectations and Chapter 5 added to this idea of resistance by examining how the SIL program has structured it. To resist a protocol, caseworkers or clients went outside the normal procedures in attempt to make life less stringent.

An example of individual resistance was when a client, Donique, passed talking to her caseworker and went straight to the administrator to advocate for herself. She desired to go to school but had no childcare and insisted on talking to someone in a position of power. Donique wrote a letter requesting childcare and used the office's fax machine to send it to the administrators. The administrators reprimanded the caseworkers for allowing her to use the fax machine. They did not want to take responsibility in denying her childcare and blocked all means of communication with Donique. This program required the adolescents to make adult decisions, but when Donique advocated for herself, her requests went ignored. Taking the information directly to the administrators did not prompt a change in the protocol on childcare.

There was collective resistance at the Sistahs' Event, where a health provider came to educate them. This was out of the ordinary because these mothers rarely gathered for social events and typically were stereotyped against. Some of these issues were being discussed at the event when the girls felt Janice, a live-in staff member, was being disrespectful by instructing them to no longer congregate in front of their building due to non-SIL residents complaints. In retaliation, they challenged and laughed at how powerless Janice was in enforcing the policies. Collective resistance was not common, but the girls did not stand for "onesided surveillance." The resistance here, though a collected effort, was weak and could not produce governmental shifts.

System Kids argued that government system leaders need to be on the ground to fully understand the magnitude of stress they place on these mothers. It showed how the system contradicts itself and explored unhealthy practices. Forcing youth to be adults, when they may not be capable of making those types of decisions, results in traumatic stress and can eventually lead to failure. This book revealed information that should be strongly considered because it would reach out to the community and authority figures who hold these stigmas and negative perceptions of these adolescents. Breaking down these stigmas and showing others that these adolescents do not always bring this stress upon themselves, but rather that the system does, would allow young mothers to identify themselves differently. Adolescence marks a critical time in development when young people are learning to juggle life while becoming their own being. Silver illustrated every narrative effectively and allowed the reader to empathize with the clients without negating the frustrations of the staff. This study presented anecdotal accounts that defined the daily hardships that young mothers face, eroded the preconceived notions of their circumstances, and challenged the quality of the services provided by the government. Implementing the practical points made throughout this book would go a long way toward positively impacting programs similar to SIL nationwide.

Conflict of interest None.

\section{Reference}

Silver, L. J. (2015). System kids: Adolescent mothers and the politics of regulation. Chapel Hill, NC: The University of North Carolina Press. 